\title{
Research and Design of IOT Gateway
}

\author{
Dawei $\mathrm{Xu}^{1, \text { a }}$, LiPing Yang ${ }^{2, \mathrm{~b}}$, Li Jiang ${ }^{1, c}$ \\ ${ }^{1}$ School of Electronic and Information Engineering, Changchun University, Changchun, 130022, \\ China \\ ${ }^{2}$ College of Computer Science and Technology, Changchun University, Changchun, China \\ aemail: 24003908@qq.com, bemail: 648824133@qq.com ,cemail: 53042520@qq.com
}

\section{Keywords: Internet of Things; Gateway; Sensor Networks}

\begin{abstract}
Things gateway can perceive protocol conversion between networks and communications networks and different types of networks, both wide-area connectivity, local interconnect can be achieved. Research and design of Things embedded gateway implementations, is to build the entire architecture of Things crucial step. Gateway is built on top of the network layer protocol converters, and embedded systems with a dedicated and strong, real-time, high reliability, low power consumption, small size and low cost, thus greatly increasing the use of embedded gateway application flexibility and reduces costs.
\end{abstract}

\section{IOT Architecture}

Things layered network communication system architecture, including the perception of extension systems, transmission systems, operations management systems and applications, supporting different communication protocols on different levels. Perception extension system includes sensing and control technology, as well as by the perception extends layer device gateways support a variety of network -aware extension includes Lonworks, UPnP, Zigbee and other communication protocols, including[1]. Sensing devices can be connected by a variety of access technologies to a core network, remote transmission of data. Operations management system for the facility within the scope of things, including the applications and business management support systems [2]. Application metering system provides statistics for end users, remote monitoring and control, intelligent interaction and other types of business expansion. Business management support system to achieve user management, security, authentication, authorization, and accounting functions.

\section{IOT Gateway Hierarchy}

Things gateway supports multiple communication protocols perception extends between devices and data types, to achieve a variety of sensing devices convert data communication between an extended formats for uploading unified data format, while the lower reaches of the acquisition or extension of the network -aware control commands are mapped to produce messages that meet specific device communication protocol. Things gateway device perception extends unified control and management , to the upper shield the underlying heterogeneity of perception extend the network is divided into four layers, namely, the business service layer, standard message constitute layer protocol adaptation layer and extending layer perception.

\section{A. Business Services layer}

And a message sending module receiving module consists message. Standard message receiving module receives a message from IOT operations management system, will deliver the message to the standard message of the constituent layers. Messaging module is responsible for the operations management system to reliably deliver sensing data collected extended network. This layer receives the message format and the message sent must meet the standards [3].

\section{B. Standard message constituent layers}

By the message parsing module and a message conversion module. Standard message parsing module parse messages from the business services layer, call the message conversion module 
converts the standard message underlying perception depends on the specific device data communication protocol format extension devices can understand. When the sensing layer extends to upload data, the message parsing module layer parsing depends on the specific device communication protocol of the message, invoke message conversion module to convert it to a message can be received by the business service layer standard format . News of the constituent layers are the core networking gateway, complete the standard message and extension of the network depends on the particular perception of the message parsing, and achieve mutual conversion between the two, to achieve a unified control and management of the underlying perception of extended networks up shielding the underlying network the purpose of the communication protocol heterogeneity [4].

\section{Protocol adaptation layer}

Protocol adaptation layer to ensure that different perception extends layer protocol can become a unified data format and control signaling through this layer [5].

\section{Perception extends Layer}

This level extend to the underlying sensing apparatus comprising message sending and message receiving two sub-modules. Message sending module is responsible for the message through the constituent layers can be converted is transmitted message specific sensing device appreciated extending to the underlying device. Message receiving module receives the message from the bottom of the device, a message is sent to the standard constituent layers parsed. Extension of a network of sensing devices sensing, including shot RFID, GPS, video surveillance systems, various types of sensors. Support for multiple communication protocols between perceptions extends equipment, can be composed of Lonworks and Zigbee and many other network -aware extension [6].

\section{IOT Gateway Design}

\section{A. Hardware structure}

The overall structure of the gateway is shown in Fig. 1 , the data acquisition module for acquisition of data or aggregation of physical world, it may be a sensor network sink node , RFID network of readers, video capture devices, GPS and so on . Processing / storage module is the core module of the gateway, which implements the protocol conversion, management, security and other aspects of data processing and storage. Access module WAN gateway access, possible ways include using a wired (Ethernet, ADSL, FTT, etc.), wireless (WLAN, GPRS, 3G, satellite, etc.). Power supply module is responsible for the gateway; the power supply may include electricity, solar, battery and so on.

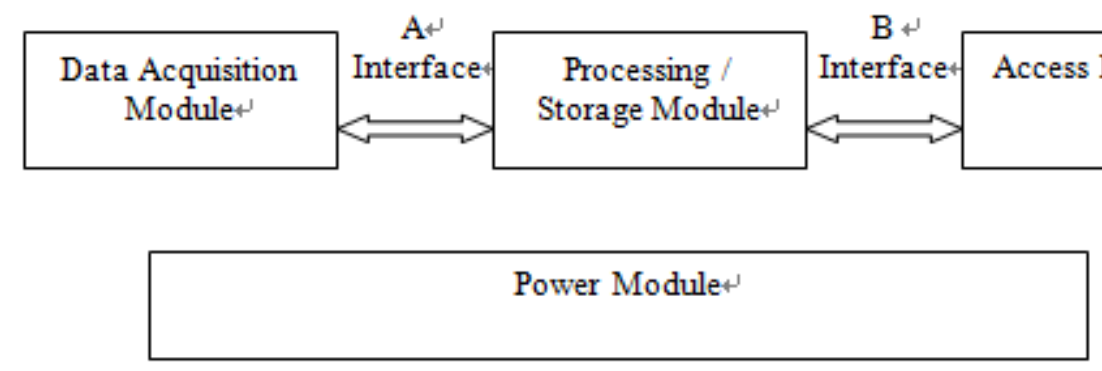

Fig.1. Gateway overall structure

The interface between the data acquisition module and processing / storage module called an interface, the interface between the access module and processing / storage module called B interface. Type A connector may have UART ( most used way ), RS232 / RS485 ( such as a variety of instruments, meters ), SPI ( direct short -range RF modules operate with ), USB ( video head ), Ethernet, AD bus, etc ; B type interface may have UART, USB, AD bus and so on. During the development process needs to define the norms and standards gradually interfaces A and B interface.

\section{B. Software Architecture}

Gateway is based on a modular design concept means to achieve the various parts of the hardware and software, making between modules is very easy to replace. Wherein the hardware module using 
the bus in the form (e.g. UART, USB, PCI, Local Bus, etc.) are connected, the software can be loaded by way of a modular operation, and abstract as a common module common portion, as shown in Figure 2. Therefore, support for new data aggregation modules and access modules only need to develop appropriate hardware modules and driver. In addition, the processing of data in the process of a unified, partial load using TLV (Type, Length, Value) approach to the organization .

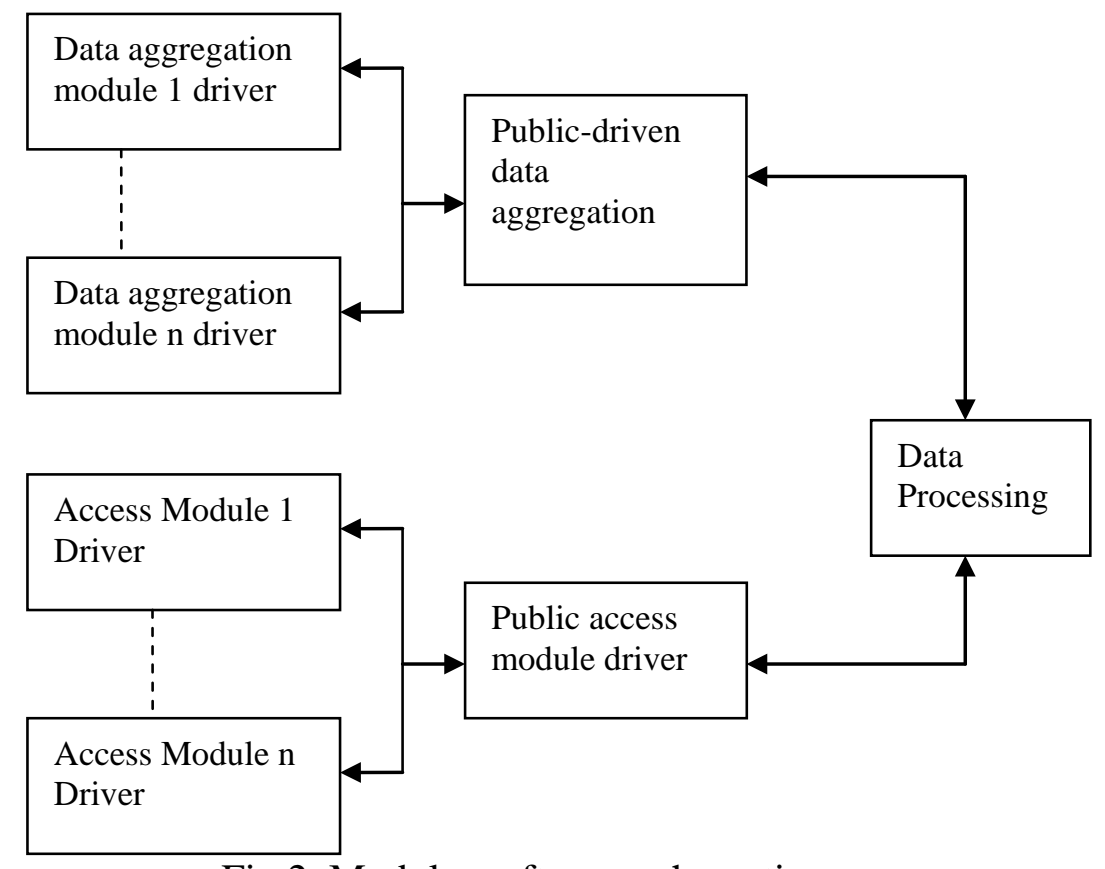

Fig.2. Modular software schematic

\section{IOT Gateway Implementations}

DEMO present need to develop an overall configuration system, wireless sensor nodes collect information such as ambient temperature, over a wireless multi-hop self-organization to send data to the gateway, the stationary reader to read the RFID tag content to the gateway; gateway these data send via WCDMA network to a server; server data processing, storage, and provides an information platform for the user (including PC users and mobile phone users) to use. By implementing this DEMO system, the key technology of things related research.

\section{A. Hardware}

1. RFID Tags: RFID tags select the card, and demonstrate the use of convenience.

2. Fixed Reader: stationary reader choose inexpensive card reader function only ordinary serial reader.

3 wireless sensor nodes: wireless sensor node selection is currently supporting the IEEE 802.15.4 standard, ZigBee and 6LoWPAN node can support the agreement.

\section{B. System Architecture}

DEMO system gateway architecture shown in Figure 3, the fixed reader with USB and wireless

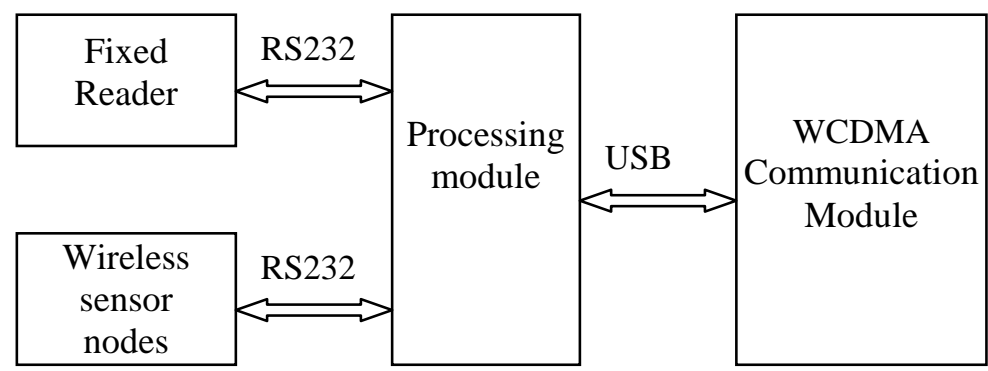

Fig.3. System Gateway Chart

sensor node processing modules communicate through RS232 communication with processing module, WCDMA communication module.

Processing module is currently considering two ways: 
(1) Using the PC as a processing module.

(2) Using the ARM processor processing module. The server uses a PC to office.

\section{Software Components}

1. The wireless sensor node software: software -based wireless sensor nodes microcontroller and C language development, the purchase of wireless sensor nodes based on the code can be done quickly.

2. Gateway Software: gateway software developed using the Linux operating system. The lowest level for the hardware drivers, protocol conversion in the application, configuration management and other applications.

Need to implement features include: protocol conversion between wireless sensor networks and RFID network and WCDMA networks, where the main consideration is among network packets organization and conversion;

Configuration Management: Using the Console, Telnet, Web are several ways to configure the gateway;

\section{Server Software}

The server software includes a communication module, a data processing module, Web / UI module and a storage module. Communication module is responsible for receiving data, the data processing responsible for the data Web / UI generated by the pack or unpacks the received data stored in the database.

\section{Conclusion}

Things gateway plays a key role in the IOT. This paper aims to study a variety of gateway devices to support heterogeneous sensor networks and access networks that can support different types of sensor nodes ( e.g. wireless ZigBee, 6LoWPAN , etc., wired as RS485, CAN , etc. ) and access methods (if line , WLAN, GPRS, 3G , etc. ), and can provide a unified data format for middleware or application, so as to apply the screen different sensor networks and access networks , making the application only need to focus on the application environment data deal with. Things gateway research in the next step will integrated firewall, VPN, DoS, traffic management, IPS, IDS, online behavior management, content filtering, WEB security , anti-virus, anti-spam and other functional modules to meet a wide protection needs, in order to realize the three-dimensional full- service security guarantee.

\section{References}

[1] Kumar, S.Budin, E.M. Prevention and management of product recalls in the processed food industry: a case study based on an explorer's perspective [J]. Techinnovation, 2006, 26: 739-750

[2] Neil Vass, M.D. Tracking weakest links in cold chain [J]. 2006

[3] Diogo M., Souza-Monteiro, Julie A.Caswell.The Economics of Implementing Traceability in Beef Supply Chains: Trends in Major Producing and Trading Countries [D].Working paper, University of Massachusetts Amherst, 2004

[4] Aruoma, O.I. The impact of food regulation on the food supply chain [J]. Toxicology, 2006, 221: 119-127

[5] K.Finkenzeller, RFID Handbook. Fundamentals and applications in contactless smart cards and identification, Wiley, Chichester, England, 2003

[6] Bill Glover, Himanshu Bhatt, RFID essentials, O'Reilly Media, Inc., 2006 ISBN 0-596-00944-5, P88-89 